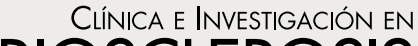 ARTERIOSCLEROSIS
}

Sociedad

Española de

Arteriosclerosis www.elsevier.es/arterio

ARTÍCULO ESPECIAL

\section{Epidemiología de la diabetes mellitus en Sudamérica: la experiencia de Colombia}

\author{
Hernando Vargas-Uricoechea ${ }^{\mathrm{a}, *}$ y Luz Ángela Casas-Figueroa ${ }^{\mathrm{b}}$ \\ a División de Endocrinología y Metabolismo, Departamento de Medicina Interna, Universidad del Cauca, Popayán, Cauca, Colombia \\ b División de Endocrinología, Fundación Clínica Valle de Lili, Universidad CES e ICESI, Cali, Valle del Cauca, Colombia
}

Recibido el 9 de noviembre de 2015; aceptado el 9 de diciembre de 2015

Disponible en Internet el 11 de mayo de 2016

\section{PALABRAS CLAVE \\ Diabetes; \\ Epidemiología; \\ Prevalencia; \\ Incidencia; \\ Carga global; \\ Hiperglucemia}

\section{KEYWORDS}

Diabetes;

Epidemiology;

Prevalence;

Incidence;

Global burden;

Hyperglycaemia
Resumen Para la Federación Internacional de Diabetes, el 8,3\% de la población mundial padece diabetes mellitus, y se espera que el número de individuos con la enfermedad se incremente a más de 592 millones. Para Sudamérica y Centroamérica se calcula que el aumento en el número de casos diagnosticados en el periodo de tiempo desde el año 2013 al año 2035 sea del 59,8\% (pasando de 24 a 38,5 millones). Para la Organización Mundial de la Salud, en el año 2014 y para la región de las Américas la prevalencia de hiperglucemia en ayunas fue del 9,3\% en hombres y del $8,1 \%$ en mujeres, y los países con mayor prevalencia de diabetes mellitus en adultos $\geq 18$ años fueron: Guyana, Surinam, Chile y Argentina. En Colombia, la prevalencia de diabetes mellitus tipo 2 es variable, dependiendo del rango poblacional evaluado y del criterio diagnóstico empleado.

( 2016 Sociedad Española de Arteriosclerosis. Publicado por Elsevier España, S.L.U. Todos los derechos reservados.

\section{Epidemiology of diabetes mellitus in South America: The experience of Colombia}

Abstract According to the International Diabetes Federation, 8.3\% of the world population suffers from diabetes mellitus, and it is expected that the number of individuals with the disease will increase to over 592 million. In South and Central America, it is estimated that the increase in the number of cases diagnosed in the period from 2013 to 2035 will be $59.8 \%$ (from 24 to 38.5 millions). According to the World Health Organisation, the prevalence of fasting hyperglycaemia in the region of the Americas in 2014 was $9.3 \%$ in men and $8.1 \%$ in women. The countries

\footnotetext{
* Autor para correspondencia.

Correos electrónicos: hernandovargasuricoechea@gmail.com, hernandovargasu10@yahoo.com (H. Vargas-Uricoechea).
} 
with the highest prevalence of diabetes mellitus in adults $\geq 18$ years were: Guyana, Surinam, Chile, and Argentina. In Colombia, the prevalence of type 2 diabetes mellitus is variable, depending on the population range assessed and the diagnostic criteria used.

(c) 2016 Sociedad Española de Arteriosclerosis. Published by Elsevier España, S.L.U. All rights reserved.

\section{Introducción}

La frecuencia de la diabetes mellitus (DM) se ha incrementado de manera global, originando una enorme carga en el aspecto social, económico y en salud. Es considerada una de las enfermedades que mayores cambios causa en los sistemas de salud de todas las naciones, independientemente del nivel de ingresos y del estado socioeconómico. La Federación Internacional de Diabetes (IDF) establece, en la sexta edición de su atlas, que el $8,3 \%$ de la población mundial padece DM (382 millones de personas), y se proyecta que esta cifra se incremente a más de 592 millones de casos en menos de 25 años, con un aumento del $55 \%$ (teniendo en cuenta, además, que hay al menos 175 millones de personas con la enfermedad y que no han sido diagnosticadas). El mayor número de casos lo aporta la región del Pacífico Occidental, con 138 millones, seguido por el Sudeste de Asia (72 millones), Europa (56 millones), Norteamérica y el Caribe (37 millones), el Medio-este y el Norte de África (35 millones); Sudamérica y Centroamérica (SACA) (24 millones) y África (20 millones). Se calcula que el aumento en el número de casos diagnosticados para SACA en el periodo de tiempo desde el año 2013 al año 2035 sea del 59,8\% (pasando de 24 a 38,5 millones). Los países con mayor número de personas con DM son (en rango de edad de 20-79 años, estimados en el año 2013): China (98,4 millones), India (65,1 millones) y Estados Unidos (24,4 millones); en Latinoamérica aparecen Brasil (11,9 millones) y México (8,7 millones) como los dos países que mayor aporte hacen al global de personas con el diagnóstico. Para la Organización Mundial de la Salud (OMS) en Sudamérica, en el año 2014, la prevalencia de DM en adultos $\geq 18$ años, según el país evaluado y de acuerdo al género (hombre-mujer, respectivamente) fue: Argentina $(\mathrm{H}: 9,9 \%$, M: 8,2\%); Bolivia (H: 6,7, M: 8,5\%); Brasil (H: 8,5, M: 7,2); Chile (H: 10,6, M: 9,5); Colombia (H: 8,5, M: 8,5); Ecuador $(H: 7,9, M: 8,5)$; Paraguay $(H: 7,6, M: 7,2)$; Perú $(H: 7,8, M:$ 8,5); Uruguay $(H: 9,5, M: 8,5)$; Venezuela $(H: 9,7, M: 8,4)$.

En Colombia, la prevalencia de DM tipo 2 (DM2) es variable, dependiendo del rango poblacional evaluado, del criterio diagnóstico empleado y de la población específica en la cual se aplican los criterios diagnósticos. En esta región de Sudamérica el número de individuos con DM2, según la IDF, es de 2.135.380. La prevalencia de DM tipo 1 (DM1) estimada es del $0,07 \%^{1,2}$.

En la sexta edición del Atlas de la IDF, la prevalencia de DM e intolerancia a la glucosa (ITG) fue estimada para los años 2013 y 2035, a partir de los datos suministrados de 219 países y territorios (agrupados en las 7 regiones de la IDF: África, Europa, Medio-este y Norte de África, Norteamérica y el Caribe, SACA, Sudeste de Asia y Pacífico Occidental).

Para la IDF, la realización de cálculos mundiales sobre la carga de DM y de alteración en la tolerancia a la glucosa depende en gran parte de la disponibilidad de datos procedentes de estudios y encuestas llevados a cabo en comunidades de todo el mundo. Dichos datos crudos proporcionan la base para los cálculos de los modelos a escala nacional y mundial. Los datos utilizados proceden de distintas fuentes, como la literatura revisada por pares, encuestas nacionales y regionales sobre salud, comunicaciones personales aportadas por investigadores de la red de la IDF y de los informes oficiales de organizaciones multinacionales, como las Naciones Unidas o los Centros para el Control y la Prevención de Enfermedades estadounidense. Los cálculos para la DM en adultos tienen en cuenta el porcentaje de personas que viven en áreas urbanas y también corrige con base en el número de casos estimados no diagnosticados. Las proyecciones tienen en cuenta los cambios de la estructura de la población y de la urbanización, pero no incluyen explícitamente cambios en la prevalencia de factores de riesgo de DM. Cuando no existen datos disponibles para un país en concreto, se utiliza la información de otros países ajustada por origen étnico, nivel de ingresos y geografía. De acuerdo con lo anterior, para la IDF la región de SACA incluye 20 países y territorios, todos con un perfil similar de distribución por edad y con cerca del $14 \%$ de la población con una edad estimada mayor de 50 años (con un incremento esperado hasta del $25 \%$ para el año 2035). El número de personas con DM para la región fue de 24,1 millones $(8,0 \%$ de la población adulta); además, este número se espera que aumente a cerca de 38,5 millones para el año 2035; adicionalmente se calculan 22,4 millones de personas $(7,4 \%$ de la población adulta) con ITG (tablas 1 y 2). Solo en Sudamérica, Brasil documentó 11,9 millones de personas con DM, Colombia 2,1 millones, Argentina 1,6 millones y Chile 1,3 millones. Finalmente, se estimó una cifra de 7.300 niños

Tabla 1 Prevalencia de intolerancia a la glucosa según la IDF 2013 (Sudamérica)

\begin{tabular}{lll}
\hline Zona geográfica & Edad (20-79 años) & $\begin{array}{l}\text { Prevalencia } \\
\text { nacional } \\
\text { Países de Sudamérica }\end{array}$ \\
\hline Argentina & Número de casos & 8,71 \\
Bolivia & 2.342 .640 & 7,19 \\
Brasil & 412.940 & 6,18 \\
Chile & 8.151 .570 & 8,94 \\
Colombia & 1.081 .780 & 8,36 \\
Ecuador & 2.506 .440 & 8,15 \\
Paraguay & 760.580 & 8,72 \\
Perú & 334.650 & 8,11 \\
Uruguay & 1.489 .480 & 9,14 \\
Venezuela & 207.290 & 8,25 \\
Guyana & 1.537 .790 & 11,02 \\
Surinam & 47.080 & 11,76 \\
\hline
\end{tabular}


Tabla 2 Prevalencia de la diabetes mellitus (DM) según la OMS 2015 y la IDF 2013 (Sudamérica)

\begin{tabular}{|c|c|c|c|c|c|}
\hline \multicolumn{3}{|c|}{ Prevalencia de DM según la OMS (2015) } & \multicolumn{3}{|c|}{$\begin{array}{l}\text { Número de hombres y mujeres con DM y } \\
\text { prevalencia nacional IDF (2013) }\end{array}$} \\
\hline Zona geográfica & $\begin{array}{l}\text { Prevalencia } \\
\text { en hombres }\end{array}$ & $\begin{array}{l}\text { Prevalencia } \\
\text { en mujeres }\end{array}$ & $\begin{array}{l}\text { Número de } \\
\text { hombres con } \\
\text { DM }\end{array}$ & $\begin{array}{l}\text { Número de } \\
\text { mujeres con } \\
\text { DM }\end{array}$ & $\begin{array}{l}\text { Prevalencia } \\
\text { nacional }\end{array}$ \\
\hline Países de Sudamérica & $(\%)$ & $(\%)$ & Edad: $20-79$ años & Edad: $20-79$ años & Edad: $20-79$ años \\
\hline Argentina & 9,9 & 8,2 & 759.160 & 848.640 & 5,98 \\
\hline Bolivia & 6,7 & 8,5 & 166.540 & 194.540 & 6,29 \\
\hline Brasil & 8,5 & 7,2 & 5.778 .010 & 6.155 .560 & 9,04 \\
\hline Chile & 10,6 & 9,5 & 560.150 & 693.800 & 10,36 \\
\hline Colombia & 8,5 & 8,5 & 1.097 .350 & 1.038 .030 & 7,12 \\
\hline Ecuador & 7,9 & 8,5 & 260.670 & 269.460 & 5,68 \\
\hline Paraguay & 7,6 & 7,2 & 111.010 & 125.800 & $6,17^{b}$ \\
\hline Perú & 7,8 & 8,5 & 387.350 & 398.910 & 4,28 \\
\hline Uruguay & 9,5 & 8,5 & 66.460 & 77.360 & $6,34^{b}$ \\
\hline Venezuela & 9,7 & 8,4 & 604.400 & 627.640 & 6,61 \\
\hline Guyana & 10,6 & 13,1 & 18.170 & 41.980 & $14,08^{b}$ \\
\hline Surinam & 11,5 & 12,5 & 16.980 & 19.770 & $10,87^{b}$ \\
\hline
\end{tabular}

a Se definió como un valor de glucemia basal $\geq 126 \mathrm{mg} / \mathrm{dl}(7,0 \mathrm{mmol} / \mathrm{l})$, o el uso de medicamentos para el manejo de la hiperglucemia.

b Prevalencia estimada de DM, basada en la extrapolación de países similares.

con DM1 en el año 2013, pero en el total de la región se estimó que 45.600 niños menores de 15 años tenían DM1 (la mayoría de ellos -es decir, 31.100- viven en Brasil). Con relación a la mortalidad, en 2013 murieron 226.000 adultos por DM (lo cual constituye el $11,6 \%$ de todas las muertes ocurridas en la región), y más del $56 \%$ de dichas muertes ocurrieron en personas mayores de 60 años: la mayoría en hombres (121.000 muertes), con 105.000 en mujeres. Por su parte, Brasil aportó en total 122.000 muertes (más de la mitad de muertes por DM para la región). Para el año 2014 la IDF actualizó nuevamente sus datos, estableciendo que para una población total de 481 millones (y una población adulta de 305,6 millones) se estimó un número de personas con DM (rango de edad de 20-79 años) de 24,8 millones, para una prevalencia regional del $8,1 \%$ (por lo que se establece que una de cada 12 personas en SACA tiene DM), con 6,8 millones de casos no diagnosticados y 219.100 muertes relacionadas con DM (con un $45,6 \%$ de dichas muertes ocurridas en personas menores de 60 años). Finalmente, el gasto sanitario total relacionado con DM fue de 28.700 millones de US\$. Los países con el mayor número de personas con DM en orden descendente fueron: Brasil, Colombia, Argentina, Chile y Venezuela (tabla 3$)^{2,3}$.

Recientemente, la OMS realizó una compilación de datos de sus 194 Estados miembros, e incluyó un resumen de los progresos tendentes a alcanzar las metas de desarrollo del milenio relacionadas con la salud y objetivos asociados. Los indicadores utilizados en dicho reporte fueron incluidos sobre la base de su relevancia a la salud pública global, a la disponibilidad, calidad, confiabilidad y comparabilidad de los resultados estimados, y tomados en conjunto suministraron un resumen de los sistemas nacionales de salud ${ }^{2}$. No obstante, ante la debilidad y la pobre calidad de los sistemas de información y estadísticas en salud en muchos países, se realizaron esfuerzos adicionales con el fin de asegurar el mejor uso de los datos reportados por cada país y poder maximizar la comparabilidad de las estadísticas entre los diferentes países (sin embargo, algunos indicadores reportados presentaron un grado de incertidumbre significativo). Se definió DM como el porcentaje de la población $\geq 18$ años con glucemia en ayunas $\geq 126 \mathrm{mg} / \mathrm{dl}$ (o bajo tratamiento específico para hiperglucemia). Para la región de las Américas (35 países comprendidos en Norte, Centro y Sudamérica), la prevalencia de hiperglucemia en ayunas fue del $9,3 \%$ en hombres y del $8,1 \%$ en mujeres (tabla 1 ).

A pesar de los datos suministrados por la IDF y la OMS, existen datos regionales y nacionales en los diferentes países de Sudamérica que en ocasiones arrojan resultados diferentes a los reportados por IDF-OMS, lo cual puede estar explicado por el tipo de definición utilizada para el diagnóstico de DM, ITG y prediabetes en general -incluyendo el diagnóstico de glucemia alterada en ayunas (GAA)- y por el hecho de que algunos de los estudios publicados en la región toman como criterio diagnóstico el uso de antidiabéticos orales y el autorreporte; además, la IDF estipula la frecuencia de DM en adultos de 20-79 años, pero en algunos estudios regionales se define el punto de corte de edad adulta como una edad $\geq 18$ años (criterio que aplica la OMS, por ejemplo, pero no la IDF). Finalmente, existen informes publicados en población $\geq 80$ años (población que no toma en cuenta la IDF). Dichos resultados también se describen en esta revisión.

\section{Distribución demográfica de Sudamérica}

Sudamérica (también denominada América del Sur o Suramérica) es el subcontinente austral de América. Es una tierra de contrastes y extremos geográficos, demográficos y culturales. Está atravesada por la línea ecuatorial en su extremo norte, quedando por lo tanto con la mayor parte de su territorio comprendida dentro del hemisferio sur. Se sitúa entre el océano Atlántico y el océano Pacífico, y ocupa una superficie de $17.819 .000 \mathrm{~km}^{2}$, lo que representa 
Tabla 3 Muertes relacionadas por diabetes mellitus (DM) y costo promedio 2013 y 2014, y prevalencia nacional de DM 2014, según IDF (Sudamérica)

\begin{tabular}{|c|c|c|c|c|c|}
\hline \multicolumn{3}{|c|}{$\begin{array}{c}\text { Muertes relacionadas con DM y costo promedio } \\
\text { por persona según la IDF (2013) }\end{array}$} & \multicolumn{3}{|c|}{$\begin{array}{l}\text { Prevalencia nacional de DM, muertes relacionadas, y } \\
\text { costo promedio por persona, según la IDF (2014) }\end{array}$} \\
\hline Zona geográfica & Edad (20-79 años) & $\begin{array}{l}\text { Costo } \\
\text { promedio } \\
\text { por persona }\end{array}$ & $\begin{array}{l}\text { Prevalencia } \\
\text { nacional (20-79 } \\
\text { años) }\end{array}$ & $\begin{array}{l}\text { Edad (20-79 } \\
\text { años) }\end{array}$ & $\begin{array}{l}\text { Costo promedio } \\
\text { por persona }\end{array}$ \\
\hline Países de Sudamérica & $\begin{array}{l}\text { Muertes } \\
\text { relacionadas con } \\
\text { DM (número) }\end{array}$ & US\$ & $\%$ & $\begin{array}{l}\text { Muertes } \\
\text { relacionadas con } \\
\text { DM (número) }\end{array}$ & US\$ \\
\hline Argentina & 15.328 & 1.174 & 6,0 & 15.221 & $1.422,7$ \\
\hline Bolivia & 5.260 & 185 & 6,3 & 4.694 & 252,1 \\
\hline Brasil & 124.687 & 1.477 & 8,7 & 116.383 & $1.527,6$ \\
\hline Chile & 8.473 & 1.320 & 12,3 & 8.956 & 1.427 \\
\hline Colombia & 15.373 & 606 & 7,2 & 14.168 & 805 \\
\hline Ecuador & 4.539 & 476 & 5,7 & 4.541 & 562,5 \\
\hline Paraguay & 2.243 & 545 & 6,2 & 2.242 & 658,2 \\
\hline Perú & 5.407 & 426 & 6,1 & 7.650 & 523,5 \\
\hline Uruguay & 1.004 & 1.358 & 6,6 & 1.040 & $1.742,1$ \\
\hline Venezuela & 9.966 & 808 & 6,6 & 9.778 & 935,5 \\
\hline Guyana & 1.098 & 13,1 & 14,3 & 1.025 & 372 \\
\hline Surinam & 471 & 617 & 10,7 & 463 & 762,5 \\
\hline
\end{tabular}

un $42 \%$ del continente americano y un $12 \%$ de las tierras emergidas; se considera que está habitada por el $6 \%$ de la población mundial. Para el año 2014 se estimó una población de 405.938.366 habitantes (distribuida en 12 países, de la siguiente manera: Brasil: 202.656.784; Colombia: 46.245.296; Argentina: 43.024.376; Perú: 30.147.936; Venezuela: 28.868.486; Chile: 17.363.894; Ecuador: 15.654.411; Bolivia: 10.631.486; Paraguay: 6.703.860; Uruguay: 3.332.972; Guyana: 735.554, y Surinam: 573.311). Los puntos extremos continentales son: extremo norte: Punta Gallinas, en Colombia; extremo sur: Cabo Froward, en la península Brunswick, Chile; extremo occidental, punto más al oeste: Punta Pariñas o Punta Balcones, Perú; extremo oriental: Punta de Seixas, Brasil. Sudamérica concentra el $5,4 \%$ de la población mundial, y es el territorio continental de menor densidad después de Oceanía. Las mayores concentraciones humanas se localizan en la costa atlántica y en las costas venezolanas y colombianas, y son evidentes los contrastes demográficos entre las grandes ciudades que superan los 10 millones de habitantes (por ejemplo, Sao Paulo y Buenos Aires) y los extensos vacíos de población, como la región Amazónica y la Patagonia.

Desde el punto de vista étnico, Sudamérica es uno de los subcontinentes más diversos del mundo -como consecuencia de la colonización española y portuguesa- sobre una base poblacional de numerosos pueblos indígenas, y por la inmigración forzosa de esclavos desde África, sumada a la inmigración masiva de europeos y asiáticos desde el siglo xIx, y finalmente por la mezcla entre estos distintos grupos. Actualmente se estima que el número de población indígena es de al menos 20 millones de personas, de los cuales alrededor de 10 millones se encuentran en la Sudamérica andina, y otro tanto en la Sudamérica extraandina. Países como Argentina y Uruguay poseen un importante componente europeo en sus poblaciones (calculando que puede ser mayor del $80 \%$ del total), seguido por Chile (52\%) y Brasil (48\%). El país donde el porcentaje de amerindios aporta el mayor componente de la población es Bolivia, aunque existen de igual forma comunidades indígenas en Perú y Ecuador, con algunas minorías aisladas en Brasil ${ }^{4,5}$.

\section{Resultados de estudios regionales en los diferentes países de Sudamérica}

Para Perú, el Ministerio de Salud había establecido previamente unos lineamientos de políticas en el sector salud para el periodo 2007-2020; en uno de sus objetivos se priorizó la vigilancia, la prevención y el control de enfermedades como la DM. Como tal, la dirección general de epidemiología propuso un sistema de vigilancia en DM basado en aquellos casos que se atendían en los servicios de salud (y por ende, complementando los indicadores de prevalencia de la diabetes en la población general y en grupos de riesgo). Dicho sistema se había desarrollado en el año 2010 (establecido como un sistema piloto en algunos hospitales de Lima y en otras regiones del país) desarrollando un entorno virtual con notificación de casos en línea. Desde que fue implementado el inicio de la vigilancia epidemiológica, al primer semestre del 2013 se habían registrado 5.001 casos de DM en 16 hospitales (6 de ellos en Lima) y en una clínica privada de Lima, y entre enero y junio de 2013 se habían registrado 928 casos, lo cual representó el $18,6 \%$ de los casos registrados desde el inicio del estudio piloto (y el $24,2 \%$ de los casos registrados en el año 2012). El 91,1\% de los casos registrados al primer semestre del 2013 correspondieron a DM2, el 1,7\% correspondió a DM1, el 1,0\% a diabetes gestacional y el 5,8\% fueron casos en los cuales no se especificó el tipo de diabetes. Se estableció además que el $63,2 \%$ de los casos registrados (3.159 sujetos) correspondieron a mujeres y el $36,8 \%$ (1.842 sujetos) a 
hombres; el grupo de edad en donde se presentó el mayor número de casos fue el de 55-64 años ${ }^{6}$. Más recientemente se describieron los resultados de la vigilancia epidemiológica en DM en hospitales piloto (a lo largo de un año) y que pertenecían al sistema de vigilancia de DM. En este estudio transversal se documentaron las personas con DM reportada durante el año 2012 en 18 hospitales, encontrando 2.959 casos; el 91,2\% contaban con niveles de glucemia en ayunas, de los cuales el $65,4 \%$ presentaban un valor $\geq 130 \mathrm{mg} / \mathrm{dl}$. El $29,3 \%$ tenían valores reportados de hemoglobina glucosilada (HbA1c), y de ellos el 66,6\% tenían un valor $\geq 7 \%$. La complicación más frecuente fue neuropatía diabética (en el 21,4\%) y la comorbilidad más frecuente fue hipertensión arterial $(10,5 \%)$ y obesidad $(3,7 \%)$. La distribución de casos por grupo de edad demostró que el mayor número de casos se encontró en el rango de edad entre 50-69 años (para ambos sexos). El $96,8 \%$ de los casos correspondieron a DM2, el 2,5\% a DM1 y el $0,7 \%$ a diabetes gestacional ${ }^{7}$.

En Ecuador, para el año 2008 la DM fue la primera causa de muerte en mujeres (1.931 casos, para una tasa de 28 por $100.000)$ y la séptima causa de muerte en hombres (1.579 casos, para una tasa de 22,8 por 100.000$)^{8}$.

Según la Encuesta Nacional de Salud y Nutrición 20112013, se encontró una prevalencia de DM (definida como un valor de glucemia $\geq 126 \mathrm{mg} / \mathrm{dl}$ ) en la población entre 10 59 años del 2,7\% (siendo del 10,3\% para el rango de edad de 50-59 años, del 5,4\% para el rango de 40-49 años y del $1,9 \%$ para el rango de 30-39 años). Además, se encontró que la prevalencia en área urbana fue de 3,2\% y en área rural de $1,6 \%$. Esta encuesta no tuvo en cuenta el antecedente de DM, ni el hecho de recibir manejo específico con fármacos orales y/o parenterales (por lo tanto, no incluía personas con diagnóstico de DM bajo tratamiento y que tenían valores normales de glucemia al momento de la encuesta). Adicionalmente, se midió la prevalencia de resistencia a la insulina por medio del índice de Homeostasis Model Assessment (HOMA). Los puntos de corte establecidos en este estudio fueron de 3,16 (para el grupo de 10-17 años) y de 2,5 (para el grupo de 18-59 años), teniendo en cuenta que dichos puntos de corte no estaban validados para la población ecuatoriana. De acuerdo con esta definición, la prevalencia de resistencia a la insulina fue mayor del $90 \%$ en toda la población con $\mathrm{DM}$; es decir, con niveles de glucemia $\geq 125 \mathrm{mg} / \mathrm{dl}$ (siendo del $100 \%$ en el grupo de edad de 20-29 años, seguida del $96,2 \%$ en el grupo de edad de $40-49$ años). Para la población con valores de glucemia normal ( $\leq 125 \mathrm{mg} / \mathrm{dl}$ ) la prevalencia de resistencia a la insulina fue del 38,4\% (44,3\% para el grupo de edad de 40-49 años, seguido por el grupo de edad de 50-59 años, con una prevalencia del 43,2\%) ${ }^{9}$.

En Venezuela se debe tener en cuenta que la información acerca de la morbilidad del Ministerio del Poder Popular para la Salud no representa la verdadera dimensión de la situación de DM en el país, y que además no se cuenta con un sistema de vigilancia epidemiológica óptimo ni de buena calidad. A pesar de ello, la información disponible sobre prevalencia de DM entre los años 1963 y 1997 documentaba una prevalencia de DM entre 0,9-6,1\% (dependiendo del grupo de edad evaluado y del criterio diagnóstico empleado). De acuerdo con la cifra de prevalencia poblacional de DM2 (estimada entre el 5,1 y el $6,0 \%$ ), para 2010 se esperaban entre 1.470 .500 y 1.730 .000 casos. Por otra parte, los estudios hacia 1992 estimaron la prevalencia nacional en 0,5 casos por cada 100.000. Según las cifras de consultas realizadas a nivel nacional en los establecimientos de salud de predominio ambulatorio entre los años 1990 a 2008, se encontró que las tasas habían variado considerablemente (de 91,7 por 100.000 en el periodo de 1990-1992 a 422,8 en el periodo de 2005-2007, y de 399, 3 en el reporte parcial a junio de 2008). Finalmente, se encontró un incremento en las tasas de mortalidad por DM, siendo de 14,0 en 1990 y de 28,5 en 2007 (por cada 100.000) ${ }^{10}$.

En Bolivia (y asumiendo que dicho país presenta uno de los indicadores de salud con mayor deficiencia en América Latina) se dispone de datos de la prevalencia de DM a partir de un estudio de corte transversal basado en la población, donde se determinó la prevalencia de DM, hipertensión arterial, obesidad y factores de riesgo relacionados en 4 de las ciudades más grandes de Bolivia; el tamaño muestral consistió en 2.948 personas, y el diagnóstico de DM fue realizado por medio del test de tolerancia oral a la glucosa (TTOG) (carga de $75 \mathrm{~g}$ de glucosa, con medición de glucemia a las $2 \mathrm{~h}$ ) utilizando criterios de la OMS. La prevalencia total de DM fue del 7,2\%, y la de ITG, del 7,8\%; la DM fue más común entre personas de la tercera edad y en aquellas con bajo nivel de educación. Adicionalmente, el 73,1\% de los diagnosticados previamente con DM, y el $73,7 \%$ de aquellos con diagnóstico reciente, tenían sobrepeso (de acuerdo a su índice de masa corporal); el diagnóstico de hipertensión arterial se documentó en el $36,5 \%$ de las personas con DM y en el $36,6 \%$ de aquellas con DM de diagnóstico reciente (comparado con el $15,9 \%$ de la población evaluada sin DM) ${ }^{11}$.

Para el año 2002 se documentaron 1.600 muertes por DM, estableciendo una tasa de mortalidad de 19 por $100.000^{12}$.

Por otra parte, los datos que maneja el Programa de Enfermedades Crónicas No Transmisibles del Ministerio de Salud y Deportes revelan que entre 800.000 a un millón de personas padecen DM en Bolivia, lo que indica una prevalencia del $8-10 \%$, con una tasa de mortalidad de 6,3 por 100.000 habitantes ${ }^{13,14}$.

En Chile, y de acuerdo a la Encuesta Nacional de Salud 2009-2010, la prevalencia de DM2 fue del 9,4\% (8,4\% en hombres y $10,4 \%$ en mujeres; estimando por lo tanto un número de alrededor de 1.200.000 personas con DM2), lo que denota un incremento del $67 \%$ en su prevalencia, puesto que para el año 2003 la misma fue del 6,3\% (alrededor de 700.000 personas con DM2). Al evaluar la prevalencia según el nivel educativo, se encontró que en el nivel educativo bajo (de los 3 estratos educativos evaluados: bajo, medio, alto) la prevalencia alcanzó el $20,5 \%$, seguido por el nivel educativo medio y finalmente el alto $(7,1$ y $6,2 \%$, respectivamente). La mayor prevalencia se encontró en el grupo de edad $\geq 65$ años (26\%), seguido del grupo de 45-64 años (17\%); finalmente, el $52,05 \%$ recibían algún tipo de tratamiento para la enfermedad, y el 34,32\% del total de la población con DM2 tenían un adecuado control glucémico. El $88,6 \%$ de la población total reportó sedentarismo de tiempo libre; en el $25,1 \%$ se encontró presencia de obesidad, y en el $39,3 \%$, sobrepeso ${ }^{15,16}$.

Más recientemente, y con el fin de analizar los perfiles de atención y sus características asociadas en el servicio de salud de la ciudad de Concepción, se realizó un análisis descriptivo e inferencial, de tipo transversal, del estado clínico de los pacientes con DM en esta área geográfica de Chile (tomando como base la información suministrada por la Encuesta Nacional de Salud 2009-2010). De un número 
estimado de casos con DM de 38.520, en el servicio de salud de Concepción se controlaron 26.638 pacientes con DM (diagnosticada a 31 de diciembre de 2012). La distribución de los 26.638 pacientes en control, respecto a los 38.520 casos estimados según la Encuesta Nacional de Salud, determinó una cobertura global del $67,9 \%$. En total, 10.223 individuos $(38,4 \%)$ tuvieron buen control de su DM2 (HbA1c $<7,0 \%)$ y $5.542(20,8 \%)$ tenían mal control (HbA1c > 9,0\%). Finalmente, en la población de 15-64 años se documentó la mayor frecuencia de mal control glucémico (de un total de 14.809 personas con DM en este rango de edad, 3.543 estaban mal controlados, equivalente al $23,9 \%)^{17}$.

En Paraguay, para el periodo 2001-2003, de una población estimada de 16.700 .718 habitantes se documentaron 57.113 muertes (45.554 con causas definidas). La DM ocupó el tercer lugar, causando 3.340 muertes $(7,3 \%)$, con una tasa de mortalidad de 20 por 100.000 (16,6 por 100.000 en hombres y 23,5 por 100.000 en mujeres); adicionalmente, se encontró que en población indígena (1.159 individuos) la prevalencia de DM fue del 5,4\%, y la de GAA, del 7,2\%. Por otra parte, en un estudio multicéntrico no aleatorio y predominantemente urbano en 14 ciudades de diferentes regiones del país (se excluyó gran parte del Chaco) sobre la prevalencia de factores de riesgo coronario, se encontró una prevalencia de DM del $8 \%$ (con variaciones según la región estudiada entre el 5 y el $12 \%)^{18,19}$.

Según la Dirección General de Estadística, Encuestas y Censos 2012, dentro de las principales causas de enfermedades registradas en los servicios del Ministerio de Salud Pública y Bienestar Social por grupos de edad, según enfermedades (año 2010), se identificaron 14.969 casos de DM, con la mayor frecuencia en el grupo de edad de 50-59 años ( 5.518 casos) y en $\geq 60$ años $(6.146 \text { casos })^{20}$.

En Uruguay, en un estudio de factores de riesgo cardiovascular en más de 74.000 individuos (solicitantes del carné de salud en el Departamento de Clínicas Preventivas entre los años 2008 y 2011) se estimó la prevalencia de DM y prediabetes; se consideró DM ante la presencia de una glucemia $>125 \mathrm{mg} / \mathrm{dl}$ y/o el antecedente de DM, y se consideró DM controlada cuando el valor de glucemia estaba $<110 \mathrm{mg} / \mathrm{dl}$; además, se estableció el diagnóstico de prediabetes cuando el valor de glucemia estaba entre 110 y $125 \mathrm{mg} / \mathrm{dl}$. La prevalencia de DM fue del $6,8 \%(7,4 \%$ en hombres y $6,1 \%$ en mujeres). El 6,0\% de los hombres y el $25,2 \%$ de las mujeres estaban controladas. La mayor prevalencia se encontró en la población $\geq 70$ años $(20,2 \%$ en hombres y $14,3 \%$ en mujeres). La prevalencia de prediabetes fue del $11,1 \%(13,9 \%$ en hombres y $8,3 \%$ en mujeres), hallándose la mayor prevalencia en el grupo de edad de 60-69 años para ambos sexos (45\% en hombres y $59,6 \%$ en mujeres). Finalmente, la prevalencia de DM y prediabetes en la población con índice de masa corporal $(I M C) \geq 30 \mathrm{~kg} / \mathrm{m}^{2}$ fue del $13,3 \%$ (para DM) y del $18,6 \%$ (para ITG) $)^{21,22}$.

En Argentina, la Tercera Encuesta Nacional de Factores de Riesgo 2013 estableció que la prevalencia de glucemia elevada/DM en la población total fue del 9,8\% (en 2009 fue del $9,6 \%$ y en el 2005 , del $8,4 \%$ ). Adicionalmente, se documentó una baja actividad física (el 55,1\% de las personas tenían baja actividad física), el 37,1\% tenían sobrepeso y el 20,8\% tenían obesidad (estas 3 variables aumentaron con respecto a la prevalencia en el año 2009, donde la prevalencia de baja actividad física fue del $54,9 \%$, la de sobrepeso fue del $35,4 \%$ y la de obesidad, del $18,0 \%)^{23}$.

Al suponer que la incidencia de DM permanecía igual entre el periodo 2010-2020, se proyectó entonces que la cantidad de individuos con DM se incrementaría de forma controlada, por lo que para el año 2020 se estima un total de 3.469.284 diabéticos, lo cual representaría una prevalencia del $8,59 \%^{24}$.

Finalmente, para la población entre 20-85 años la carga de DM sin complicaciones en Argentina es de 1,3 millones de años de vida ajustados por discapacidad, y mientras que las tasas de mortalidad (años de vida perdidos debido a muerte prematura [YLL]) se incrementaron como una función de la edad, los años de vida vividos con discapacidad (YLD) mostraron una relación opuesta. Las mujeres tuvieron una alta carga de valores de la enfermedad (representado en el 51 y el $61 \%$ de YLL y YLD, respectivamente, e independientemente de la edad) ${ }^{25}$.

En Brasil, en el año 1992 se publicó un estudio multicéntrico que determinó la prevalencia de DM en población urbana ( 9 ciudades) en 21.847 individuos, en un rango de edad de 30-69 años, encontrando que el 7,6\% de dichos individuos tenían $\mathrm{DM}(7,5 \%$ para hombres y $7,6 \%$ para mujeres) y el 7,8\% tenían ITG (basado en el TTOG); la prevalencia de DM se incrementó del $2,7 \%$ en el grupo de edad de 30-39 años al $17,4 \%$ en el de 60-69 años (la DM fue más prevalente cuanto menor educación tenía la población, pero dicha diferencia desaparecía después de hacer el ajuste por edad). El antecedente familiar de DM se asoció con un incremento del doble en la prevalencia de DM $(12,5 \%$ en los que tenían antecedente familiar de DM versus $5,8 \%$ de los que no lo tenían); de igual forma, la misma asociación se encontró con obesidad (11,6\% para aquellos con obesidad versus 5,2\% para aquellos sin obesidad). La DM no diagnosticada se presentó en el $46 \%$ de la prevalencia total ${ }^{26}$.

Otro estudio realizado en población urbana, en 1.166 individuos entre 30-79 años, determinó la prevalencia de DM y de ITG (por medio del TTOG), encontrando que la prevalencia de DM fue del 13,5\% (11,2\% para hombres y 15\% para mujeres) y la de ITG fue del $5,0 \% 27$.

En el año 2013, el sistema de vigilancia VIGITEL (encuesta telefónica de vigilancia de factores protectores y de riesgo para enfermedades crónicas) reportó un 6,9\% de DM autorreportada; además, se encontró un incremento en la frecuencia de DM de acuerdo a la edad (8,5\% para el grupo de $45-54$ años, $17,1 \%$ en el grupo de $55-64$ años y $22,1 \%$ en el grupo $\geq 65$ años) $)^{28}$.

En general, y tomando como referencia los estudios publicados (con diferentes propósitos, tamaños muestrales y metodologías) sobre prevalencia de DM en Brasil, desde 1992 hasta 2009 (con criterios diagnósticos diferentes: autorreporte, glucemia en ayunas, TTOG y glucosa capilar en ayunas, en población adulta con diferentes estratos de edad evaluados) la prevalencia de DM osciló entre el 2,3\% (como el valor de prevalencia más bajo reportado) y el $17,9 \%$ (como el valor de prevalencia más alto reportado $)^{29}$.

Más recientemente, un estudio de cohorte fue realizado en 15.105 funcionarios seleccionados de instituciones públicas (con rango de edad entre 35-74 años) de 6 capitales de 3 regiones de Brasil. La DM fue definida por autorreporte, uso de medicamentos para DM, glucemia en ayunas, TTOG y/o HbA1c. Los puntos de corte establecidos para DM y estados 
intermedios de hiperglucemia fueron los establecidos por la OMS y la Asociación Americana de Diabetes. De acuerdo a dichos criterios, el $19,7 \%$ de los participantes se presentaron con DM (50,4\% sin diagnóstico previo). La frecuencia de estados intermedios de hiperglucemia (de acuerdo al criterio diagnóstico empleado) estuvo en el rango de 16,1-52,6\%. De acuerdo con la edad y el sexo, el mayor porcentaje de casos con DM se presentó tanto en hombres como en mujeres en el rango de edad de 65-74 años ${ }^{30}$.

En Guyana, los datos acerca de prevalencia de DM son muy escasos; en la Encuesta Demográfica y de Salud 2009, la cual fue una muestra representativa nacional, no se evaluó la frecuencia de enfermedades no transmisibles, como la DM; no obstante, la DM es la tercera causa de muerte (después de la cardiopatía isquémica y la enfermedad cerebrovascular); para el año 2008, del total de muertes registradas a nivel nacional, la DM causó el 6,2\% en hombres y el $11,4 \%$ en mujeres. Entre los años 2005 y 2008, un promedio anual de 8.872 nuevos casos de DM fueron reportados (71\% en personas menores de 65 años $)^{31}$.

En Surinam también destaca la escasez de estudios a escala nacional en la determinación de la frecuencia de DM. Las tasas de mortalidad por DM seguían siendo comparables desde 2007 (38,4 por 100.000) a 2009 (33,2 por 100.000). Un estudio de 637 pacientes con DM llevado a cabo en 12 consultorios reportó la aparición más temprana de DM en personas de ascendencia indostana (a los 44 años de edad), en comparación con las de ascendencia criolla (a los 53 años). Finalmente, los grupos étnicos que residen en las zonas urbanas tienen las tasas más elevadas de mortalidad por DM (indostanos 58,5 por 100.000; criollos 43,1 por 100.000 , y javaneses 28,8 por 100.000$)^{32-34}$.

En Colombia, los resultados disponibles pueden agruparse de la siguiente forma:

1. Datos nacionales (basados en encuestas y estadísticas gubernamentales). Para datos nacionales, se describen 3 grandes estudios. La Encuesta Nacional de Salud 2007 (ENS 2007) encontró que la prevalencia nacional de DM (autorreferida por entrevista, en población de 18-69años) fue del 3,51\%. Por su parte, la Encuesta Nacional de Demografía y Salud (año 2010) encontró una prevalencia de DM en adultos mayores del 11,2\% (por autorreporte, en población entre 60-84 años). Finalmente, en el año 2008 se creó la Cuenta de Alto Costo (CAC), cuyos datos consignados incluyen a todos los pacientes afiliados y reportados por las entidades prestadoras de salud (entre otras). Se reportaron 808.101 personas con DM, con un predominio en mujeres (480.723 casos, equivalentes al 59,49\%, mientras que en hombres el número de casos fue de 327.378 , el $40,51 \%)$. El $88 \%$ de todos los casos de DM se presentaron en personas $\geq 45$ años; el rango de edad con mayor número de casos fue el de 60-64 años (con 112.584 casos) y el rango de edad con menos casos fue el de $\leq 20$ años (con 19.807 casos). Se evidenció, además, que en Bogotá se encuentran el $20 \%$ de todos los pacientes reportados en Colombia, y que 1,84 de cada 100 personas que pertenecen al sistema general de seguridad social tienen DM (2,2 y 1,4 casos por cada 100 afiliados -en mujeres y en hombres-, respectivamente). Adicionalmente, se encontró que únicamente el 13\% de la población evaluada se encuentra controlada (con niveles de HbA1c $<7 \%)^{35-37}$.

2. Estudios de prevalencia de DM en Colombia, como parte de estudios internacionales que evalúan factores de riesgo cardiovascular. En el estudio Cardiovascular Risk Factor Multiple Evaluation in Latin America (CARMELA) la DM fue definida como un valor de glucemia en ayunas $\geq 126 \mathrm{mg} / \mathrm{dl}$ o por autorreporte; la prevalencia de DM para Bogotá (en el rango de edad entre 25-64 años) fue del $8,1 \%$, y por género fue del $7,4 \%$ para hombres y del $8,7 \%$ para mujeres. En el estudio Prospective Urban Rural Epidemiology (PURE) en individuos entre 35 y 70 años de edad, la DM fue definida por autorreporte o por la presencia de un nivel de glucemia en ayunas $\geq 126 \mathrm{mg} / \mathrm{dl}$, y según ello se encontró una prevalencia de DM del 11,9\%. Por otro lado, en el estudio del Día Internacional para la Evaluación de la Obesidad Abdominal (IDEA) en personas con rango de edad entre 18 y 80 años, la prevalencia de DM (por autorreporte) fue del 7,2\% en hombres y del $5,2 \%$ en mujeres (la prevalencia global fue del 5,8\%). Al analizar los datos de acuerdo al IMC y al género, se encontró que en hombres con IMC > 30 la prevalencia de DM se incrementaba a $13,1 \%$, y en mujeres con IMC > 30 la prevalencia se incrementaba a 10,8\% (para ambos sexos, incremento de la prevalencia de DM con IMC > 30 al compararlos con individuos con IMC $<25)^{38-42}$.

3. Estudios regionales de prevalencia de DM, «alteración en la tolerancia a la glucosa»o de hiperglucemia en ayunas. Entre 1969 y 1985 se publican estudios que abarcaron personas mayores de 15 años en población general procedentes de área urbana y/o rural (o en población exclusivamente masculina) y en personas con alto riesgo para la presencia de DM, encontrando que la prevalencia de DM oscilaba entre el 1 y el 13,2\% (dependiendo del tipo de población evaluada, los criterios diagnósticos empleados y la edad al momento del diagnóstico $)^{43-49}$. Posteriormente, entre los años 1993 y 2006 se publicaron estudios que medían la frecuencia de DM, ITG e hiperglucemia en ayunas en población > 15 años, procedentes de área urbana (con muy pocos datos que evaluaran la frecuencia en área rural), y en población especial (hipertensos y en personas con síndrome coronario agudo). La prevalencia de DM entre las diferentes poblaciones evaluadas estuvo entre 1,4-21,3\%; la de hiperglucemia en ayunas fue del $12,6 \%$, y la de ITG osciló entre 1,73-5,8\%. Finalmente, la prevalencia de GAA fue del 5,8\% (dependiendo de los criterios diagnósticos empleados y del riesgo basal de los individuos evaluados) ${ }^{50-58}$.Entre los años 2007 y 2015 se publicaron estudios en los que se encuentran resultados en población > 14 años (procedentes de área urbana y/o rural, población específica: pilotos de la aeronáutica civil, personas con VIH-sida, población exclusivamente femenina, individuos recluidos en centros penitenciarios, personas con dislipidemias y población exclusivamente indígena) que denotan una prevalencia de DM entre el 0,6 y el $28,5 \%$. La prevalencia de hiperglucemia en ayunas estuvo entre el $7 \%$ y el $45 \%$ (dependiendo de los criterios diagnósticos empleados, la edad, la procedencia, el género y el tipo de población específica evaluado). La prevalencia de ITG fue del 1,7\% para hombres y del 2,7\% para mujeres (en área urbana), y del 0,6\% para hombres y del 2,6\% para mujeres (en área rural) ${ }^{59-73}$. 
4. Estudios de prevalencia en población escolar, adolescentes e individuos menores de 20 años. Los estudios en este rango poblacional son escasos; en el año 1990 la incidencia global de DM1 en población $\leq 14$ años fue del $3,8 \%$ ( $4,7 \%$ en hombres y $2,9 \%$ en mujeres), y la prevalencia estimada fue del 1,8\%. Entre los años 2008 y 2013 se publicaron 3 estudios en población entre 0-20 años (procedentes del área urbana), encontrando una prevalencia de hiperglucemia en ayunas en población de 0-9 años del 7,9\%; para la población entre $10-18$ años la prevalencia encontrada de hiperglucemia en ayunas estuvo entre el 0,7 y el 11,3\%; para la población entre 15-20 años la prevalencia de GAA fue del $8,4 \%$, y la de ITG fue del $0,8 \%$ (dependiendo de los criterios diagnósticos empleados, de la edad y de la ocupación de la población evaluada $)^{74-78}$.

\section{Discusión}

Para la IDF 2014, los 5 países de la región con mayor número de personas con DM fueron: Brasil (11.623.000), Colombia (2.192.000), Argentina (1.626.000), Chile (1.513.000) y Venezuela (1.252.000). Además, en países como Brasil, uno de cada 12 adultos tiene DM; en Colombia, uno de cada 14; en Argentina, uno de cada 17; en Chile, uno de cada 8, y en Venezuela, uno de cada 15, y se determinó también que el número de personas no diagnosticadas (y que padecen DM) es de 3.229.000 para Brasil, de 608.900 para Colombia, de 451.700 para Argentina, de 325.500 para Chile y de 347.900 para Venezuela. Para la OMS 2015, la mayor prevalencia correspondió a Surinam, Guyana, Chile, Argentina y Venezuela (teniendo en cuenta que para la IDF, Guyana y Surinam no forman parte de SACA).

Al analizar la frecuencia de DM en Sudamérica deben tenerse en cuenta algunas consideraciones (tabla 4):

1. La forma como se realiza el diagnóstico, los criterios de identificación y cribado empleados, el tipo de población (su origen y procedencia) y el rango de edad evaluados, puesto que difieren en los diferentes estudios (nacionales, locales, regionales) y porque tampoco ha existido un criterio diagnóstico único o universal en la detección de la enfermedad (con la consecuente diferencia en los valores de prevalencia de (a DM). Lo anterior puede influir en la variación de las prevalencias descritas entre los diferentes países (y entre los mismos países).

2. La DM tiene ciertas características epidemiológicas que podrían explicar la frecuencia actual descrita para Sudamérica; por ejemplo, el hecho de ser una enfermedad de larga duración (además del hecho de que los individuos con DM usualmente se presentan en el contexto de una enfermedad que no es curable, pero que se caracteriza por tener una vida relativamente prolongada). Adicionalmente, el aumento en la incidencia, junto al fenómeno migratorio (emigración de personas sanas e inmigración de personas susceptibles), al desplazamiento forzado en algunos países y al mayor acceso a pruebas de cribado, podrían originar las diferencias regionales descritas, explicando de alguna manera el aumento en la predicción de nuevos casos de DM en los próximos años.

3. También es probable que influyan algunos factores medioambientales y socioeconómicos (transición demográfica y envejecimiento poblacional, bajo nivel educativo, transición nutricional, crecimiento económico, urbanización y aspectos sobre la promoción de la actividad física). Entre 1970 y 2011, la población mundial aumentó de 3.600 millones a 7.000 millones (y se establece que al aumentar el número total de la población, también aumenta el número de personas con enfermedades crónicas no transmisibles, como la DM). Un aspecto cada vez más preocupante es la tasa de dependencia de los países (es decir, el número de personas más jóvenes y más viejas, dividido por la población económicamente activa entre 15 y 64 años de edad), indicando que ciertas regiones podrían beneficiarse de un «dividendo demográfico», ya que la proporción de la población económicamente activa está en aumento. Entre los años 2010 y 2050 se calcula que Sudamérica tendrá alrededor de 520 millones de habitantes, con un descenso significativo en la población menor de 15 años, mientras que los individuos mayores de 65 años pasarán a representar entre el $20-25 \%$ de la población total. Esta proyección del crecimiento poblacional supone la creación de una estructura demográfica conocida como «bono demográfico», que significa una reducción de la tasa de dependencia entre las personas potencialmente inactivas (de 0 a 14 años y de 65 años y más) y las personas potencialmente productivas (de 15 a 64 años). A medida que la población envejece, se reduce la proporción de personas económicamente activas. Lo anterior estaría indicando que la población de Sudamérica, al envejecer de la forma como se ha proyectado, aportará un número importante de nuevos individuos con DM, con consecuencias negativas desde el punto de vista social y económico ${ }^{79,80}$.

4. Actualmente se calcula que la población urbana de Sudamérica es cercana al $83 \%$, lo que significa que aproximadamente 333 millones de personas viven en ciudades, considerándose una de las regiones con mayor índice de urbanización y con tendencia creciente; se estima que el porcentaje de población urbana (> 50\%) se presenta en 11

Tabla 4 Factores que pueden influir en la frecuencia de diabetes mellitus en Sudamérica

Diferencias en la forma en que se realiza el diagnóstico Transición demográfica

Envejecimiento poblacional

Crecimiento económico y bajo nivel educativo

Promoción de la actividad física

Vulnerabilidad poblacional
Desplazamiento forzoso y fenómeno migratorio Incremento en la frecuencia de obesidad y sobrepeso Malnutrición materna y niños con bajo peso al nacer Tipo y edad de la población evaluada Transición nutricional Incremento en la tasa de urbanización 
países (Argentina, Brasil, Bolivia, Chile, Colombia, Ecuador, Paraguay, Perú, Surinam, Uruguay y Venezuela), con tasas de crecimiento de hasta el 2,5\% anual. Este incremento de sus áreas urbanas (o el crecimiento de las ya existentes) sin un adecuado ordenamiento territorial se traduce en que no se pueda satisfacer la demanda de servicios básicos de la población. Adicionalmente, y según la Comisión Económica para América Latina y el Caribe (CEPAL) (2014), el crecimiento económico mundial se redujo de un 2,4\% en 2012 a un 2,1\% en 2013. La región de América Latina y el Caribe registró un crecimiento modesto, impulsado por la demanda interna; el producto interior bruto (PIB) de América Latina y el Caribe creció un 2,6\% (cifra inferior al 3,1\% registrado en 2012). Este resultado ilustra la continua desaceleración económica regional que se ha manifestado desde el año 2011, con diferencias importantes en los ritmos de crecimiento de los países. Se espera, entonces, que el alto índice de urbanización y la desaceleración económica incrementen la vulnerabilidad poblacional, con un mayor riesgo de enfermedades crónicas no transmisibles como la DM. Finalmente, factores socioeconómicos como los desplazamientos (internos y/o externos) como consecuencia de las precarias condiciones laborales, la carencia de trabajo, el pobre acceso a los servicios esenciales y educativos, la inseguridad (y en el caso de Colombia, los conflictos armados internos) pueden influir en la frecuencia de DM entre los diferentes países de la región ${ }^{81,82}$.

5. En países en vías de desarrollo, la epidemia de la obesidad y de enfermedad cardiometabólica se ha asociado con la presencia de inequidades socioeconómicas, lo que conlleva una mayor vulnerabilidad a dichas entidades, debido a la presencia de desnutrición materna (y consecuentemente hijos con bajo peso al nacer). Estos factores, junto a los cambios propios de la vida moderna, pueden modificar los hábitos dietarios y la actividad física (sedentarismo), y la adaptación de estos cambios al medioambiente puede predisponer a una ingesta excesiva de alimentos altamente calóricos que, junto al sedentarismo presente y a la interacción con otros factores, se traduce en una mayor susceptibilidad al sobrepeso/obesidad y a otros determinantes de riesgo cardiovascular. Es probable entonces que el proceso de transición de individuos con bajo peso al nacer, hijos de madres mal nutridas (y que en la vida adulta se exponen a cambios de factores medioambientales y a hábitos de vida no saludables) sean factores que conllevan al incremento en la frecuencia de obesidad y de otros factores cardiometabólicos, incluyendo DM. Teniendo en cuenta que el aumento en la prevalencia de obesidad es evidente en Sudamérica, se espera entonces un incremento en la incidencia y prevalencia de prediabetes y $\mathrm{DM}^{83,84}$.

6. La promoción de la actividad física es considerada uno de los principales pilares en la prevención de las enfermedades crónicas no transmisibles. Está claro el papel que tiene el ejercicio regular en la prevención y manejo de la DM2. La urbanización acelerada, el transporte motorizado, el incremento en el trabajo sedentario y las actividades sedentarias relacionadas con el uso de tecnologías (videojuegos, ordenador) reducen los niveles de actividad física ${ }^{85,86}$. A nivel mundial, se estima que la inactividad física origina el $27 \%$ de los casos de DM2, y se espera que dicha tendencia también se presente para Sudamérica.

\section{Conclusión}

La DM es un problema de características epidémicas en Sudamérica, y algunos factores pueden explicar su incremento y variación en la frecuencia: rápido crecimiento y envejecimiento poblacional, alto índice de urbanización, bajo nivel educativo, mayor acceso a dietas hipercalóricas y sedentarismo, entre otros. La prevalencia de la enfermedad varía entre los distintos países, con diferencias incluso dentro de ellos mismos, probablemente como consecuencia de métodos de cribado y criterios de definición diferentes, el año o periodo de realización del estudio, la población y el rango de edad evaluado, el criterio diagnóstico empleado, el origen y procedencia de los individuos y del tipo de población especial o específica evaluada. Aún existen países de la región con datos poblacionales deficientes acerca de la frecuencia de la enfermedad y sus consecuencias.

\section{Responsabilidades eticas}

Protección de personas y animales. Los autores declaran que para esta investigación no se han realizado experimentos en seres humanos ni en animales.

Confidencialidad de los datos. Los autores declaran que en este artículo no aparecen datos de pacientes.

Derecho a la privacidad y consentimiento informado. Los autores declaran que en este artículo no aparecen datos de pacientes.

\section{Conflicto de intereses}

Los autores declaran no tener ningún conflicto de intereses.

\section{Bibliografía}

1. International Diabetes Federation. The IDF Diabetes Atlas. 6th ed. Brussels: International Diabetes Federation; 2013.

2. [consultado 16 Sep 2015]. Disponible en: http://www.who.int/ gho/indicator_registry/en/

3. International Diabetes Federation. The IDF Diabetes Atlas. 6th ed. Brussels: International Diabetes Federation; 2014. Update.

4. Atlas de geografía del mundo. Primera edición, 2013. D.R. (Secretaría de Educación Pública, 2013. Argentina 28, Centro, 06020, México, D.F. ISBN 978-607-514-332-3).

5. Informe sobre Desarrollo Humano 2013. El ascenso del Sur: Progreso humano en un mundo diverso. Copyright (c) 2013 del Programa de las Naciones Unidas para el Desarrollo 1 UN Plaza, Nueva York, NY 10017, Estados Unidos.

6. Revilla L. Situación de la vigilancia de diabetes en el Perú, al I semestre de 2013. Bol Epidemiol. (Lima). 2013;22:825-8.

7. Ramos W, López T, Revilla L, More L, Huamaní M, Pozo M. Resultados de la vigilancia epidemiológica de diabetes mellitus en hospitales notificantes del Perú, 2012. Rev Peru Med Exp Salud Publica. 2014;31:9-15.

8. Lucio R, Villacrés N, Henríquez R. The Health System of Ecuador. Salud Publica Mex. 2011;53 Suppl 2:177-87. 
9. Freire WB, Ramírez MJ, Belmont P, Mendieta MJ, Silva MK, Romero N, et al. Resumen ejecutivo. Tomo I. Encuesta Nacional de Salud y Nutrición del Ecuador. ENSANUT-ECU 20112013. Primera edición Quito, Ecuador: Ministerio de Salud Pública/Instituto Nacional de Estadística y Censos; 2013.

10. Camejo M, García A, Rodríguez E, Carrizales ME, Chique J. Visión epidemiológica de la diabetes mellitus. Situación en Venezuela. Registro epidemiológico y propuesta de registro. Programas de detección precoz. Capítulo I. Rev Venez Endocrinol Metab. 2012;10 Supl. 1:1-6.

11. Barceló A, Daroca MC, Ribera R, Duarte E, Zapata A, Vohra M. Diabetes in Bolivia from Pan American Health Organization, Program on Non-Communicable Diseases, 525 Twenty-third Street, N.W., Washington, D.C. 20037-2895, USA, 2001.

12. Ledo C, Soria R. The Health System of Bolivia. Salud Publica Mex. 2011;53 Suppl 2:109-19.

13. Noa-Ávila LR, Chang-Solano M. Factores de riesgo y complicaciones en pacientes con diabetes mellitus tipo 2. Sucre. 2012;17:1-19. Multimed. 2013.

14. Abdelnur J. Diabetes en Bolivia, salud y sociedad. Ad Astra. 2014;5:53-4.

15. Encuesta Nacional de Salud 2009-2010: Ministerio de Salud de Chile [consultado 28 Oct 2015]. Disponible en: http://www.redsalud.gov.cl/portal/url/item/ 99bbf09a908d3eb8e04001011f014b49.pdf

16. Encuesta Nacional de Salud 2003: Ministerio de Salud de Chile [consultado 28 Oct 2015]. Disponible en: http://epi.minsal.cl/ estudios-y-encuestaspoblacionales/encuestas-poblacionales/ descarga-ens-2003 o en: http://epi.minsal.cl/epi/html/frames/ frame3.htm

17. Barria-von-Bischhoffshausen F, Harnisch K, Montes N, Pio G, Barría F. Análisis de los pacientes con diabetes controlados a nivel primaria en el Servicio de Salud Concepción. Rev Chil Endocrinol Diabetes. 2014;7:47-51.

18. Situación de salud de la comunidad indígena del Chaco paraguayo, 2000. Informe técnico. Asunción, Paraguay: Universidad Nacional de Asunción, Instituto de Investigación en Ciencias de la Salud; 2003

19. Salud en las Américas, 2007. Volumen II-Países (Paraguay), pp. 608-629.

20. Dirección General de Estadística, Encuestas y Censos. Anuario Estadístico del Paraguay 2012. DGEEC. Asunción, diciembre 2013 [consultado 17 Sep 2015]. Disponible en: http: //www.dgeec.gov.py

21. Fort Z, Portos A, Castro M, Piñeyro C, Ciganda C, Bermúdez Y, et al. Factores de riesgo cardiovascular en 74.420 solicitantes de carné de salud. Rev Urug Cardiol. 2012;27:150-61.

22. Presidencia de la República Oriental del Uruguay. Reporte social 2013. Principales características del Uruguay social.

23. Tercera Encuesta Nacional de factores de Riesgo para enfermedades no-transmisibles. Presentación de los principales resultados. Ministerio de Salud, Instituto Nacional de Estadística y Censos, 2013 [consultado 7 Sep 2015]. Disponible en: http://www.msal.gov.ar

24. Sánchez MA, Pagliari S, Acrogliano P, Schneider D, Belloni D. Prevalencia de la diabetes en la República Argentina: Proyecciones utilizando simulación dinámica. 39JAlIO-CAIS 2010, pp. 3057-3064.

25. González L, Caporale JE, Elgart JF, Gagliardino JJ. The burden of diabetes in Argentina. Glob J Health Sci. 2015;7:124-33.

26. Malerbi DA, Franco LJ, The Brazilian Cooperative Group on the Study of Diabetes Prevalence. Multicenter study of the prevalence of diabetes mellitus and impaired glucose tolerance in the urban Brazilian population aged 30-69 yr. Diabetes Care. 1992;15:1509-16

27. Bosi PL, Carvalho AM, Contrera D, Casale G, Pereira MA, Gronner $M F$, et al. Prevalence of diabetes and impaired glucose tolerance in the urban population of 30 to 79 years of the city of
Sao Carlos, Sao Paulo. Arq Bras Endocrinol Metabol. 2009;53: 726-32.

28. De Almeida-Pititto B, Dias ML, de Moraes AC, Ferreira SR, Franco DR, Eliaschewitz FG. Type 2 diabetes in Brazil: Epidemiology and management. Diabetes Metab Syndr Obes. 2015;8:17-28.

29. Bertoldi AD, Kanavos P, França GV, Carraro A, Tejada CA, Hallal $\mathrm{PC}$, et al. Epidemiology, management, complications and costs associated with type 2 diabetes in Brazil: A comprehensive literature review. Global Health. 2013;9:1-12.

30. Schmidt MI, Hoffmann JF, Sander Diniz MF, Lotufo PA, Griep RH, Bensenor IM, et al. High prevalence of diabetes and intermediate hyperglycemia - The Brazilian Longitudinal Study of Adult Health (ELSA-Brasil). Diabetol Metab Syndr. 2014;6:1-9.

31. Guyana. Health in the Americas, 2012 Edition. Pan American Health Organization; 2012.

32. Pan American Health Organization/World Health Organization; Suriname Ministry of Health. Crude mortality calculations based on $\mathrm{MOH}$, mortality in Suriname. Washington, DC: PAHO/WHO; Suriname: Ministry of Health; 2011.

33. Suriname, Ministry of Health. Epidemiology Surveillance Reporting. Paramaribo: Ministry of Health; 2012.

34. Suriname. Health in the Americas, 2012 Edition. Pan American Health Organization; 2012.

35. Rodríguez J, Ruiz F, Peñaloza E, Eslava J, Gómez LC, Sánchez H, et al. Encuesta Nacional de Salud 2007. Resultados Nacionales; 1.a edición. Bogotá, 2009.

36. [consultado 18 Nov 2015]. Disponible em: http://www. profamilia.org.co/encuestas/Profamilia/Profamilia/

37. Acuña-Merchán L. Situación de la enfermedad renal en Colombia 2014. Cuenta de alto costo. Bogotá, Colombia: Fondo Colombiano de Enfermedades de Alto Costo; 2015.

38. Schargrodsky H, Hernández-Hernández R, Champagne BM, Silva $H$, Vinueza R, Silva Ayçaguer LC, et al., CARMELA Study Investigators. CARMELA: Assessment of cardiovascular risk in seven Latin American cities. Am J Med. 2008;121:58-65.

39. Corsi DJ, Subramanian SV, Chow CK, McKee M, Chifamba J, Dagenais G, et al. Prospective Urban Rural Epidemiology (PURE) study: Baseline characteristics of the household sample and comparative analyses with national data in 17 countries. Am Heart J. 2013;166:636-46.

40. López-Jaramillo P. HDL y triglicéridos: ¿cuál es valor real? Recomendaciones de la Asociación Colombiana de Endocrinología, DM y Metabolismo para el manejo de la dislipidemia. Asociación Colombiana de Endocrinología, Diabetes y Metabolismo; 2013.

41. Aschner P, Ruiz A, Balkau B, Massien C, Haffner SM, On behalf of the Latin America and the Caribbean International Day for Evaluation of Abdominal Adiposity (IDEA) National Coordinators and Investigators. Association of abdominal adiposity with diabetes and cardiovascular disease in Latin America. J Clin Hypertens (Greenwich). 2009;11:769-74.

42. Ruiz AJ, Aschner PJ, Puerta MF, Alfonso-Cristancho R. Estudio IDEA (International Day for Evaluation of Abdominal Obesity): prevalencia de obesidad abdominal y factores de riesgo asociados en atención primaria en Colombia. Biomédica. 2012;32:610-6.

43. COLOMBIA. Ministerio de Salud Pública y Asociación de Facultades de Medicina. Estudio de recursos humanos para la salud y la educación médica en Colombia. Evidencia clínica. Bogotá 1969. pp. 51-53.

44. Espinosa H, Zambrano UF, González G. Enfermedad coronaria, hipertensión, DM y úlcera péptica en ejecutivos de Medellín. Ant Medica. 1975;25:253-62.

45. Velásquez JT, Espinosa de RH, Zambrano UF. Enfermedad coronaria, hipertensión, DM y úlcera péptica en ejecutivos de Medellín en consulta privada. Ant Medica. 1975;25:263-72.

46. Zambrano UF, Espinosa de RH, Molina I. Encuesta de prevalencia de DM en un barrio de la ciudad de Medellín, su asociación con hipertensión y obesidad. Ant Medica. 1975;25:521-3. 
47. Triana A, Cortés S, Hoyos N, López C, Remolina E, Rodríguez B. Encuesta de prevalencia de DM en Fresno, Tolima. IV Congreso Latinoamericano de DM, Caracas. Venezuela. 1980.

48. López C, Cortés S, Hoyos N, Triana A, Castillo D, Sánchez-Medina $M$. Encuesta de prevalencia de DM en individuos con alto riesgo en Fusagasugá, Cundinamarca. IV Congreso Latinoamericano de DM, Caracas Venezuela, 1980;41.

49. Soto C, Zuloaga L, Jaramillo DE, Robledo N, Betancur C, Duque B. Prevalencia de la alteración en la tolerancia a la glucosa. Barbosa, Antioquia. Acta Med Colomb. 1985;10: 22-33.

50. Aschner P, King H, de Torrado M, Rodríguez BM. Glucose intolerance in Colombia: A population based survey in an urban community. Diabetes Care. 1993;16:90-3.

51. Suárez-Russi MA, López de Mesa C, Urbano F, Coral A, Villota E, Poveda de Ruiz H, et al. Prevalencia de DM en la ciudad de Pasto, Nariño, Colombia. Biomédica (Bogotá). 1993;13: 187-94.

52. Aschner P, Chaves M, Izquierdo J, Sole J, Tarazona A, Pinzon $\mathrm{JB}$, et al. Prevalence of the metabolic syndrome in a rural and an urban population in Colombia. Diab Res Clin Pract. 2002;57 Suppl 1:S32. P0079 (A0096).

53. Aschner P. Síndrome metabólico en una población rural y una población urbana de la región andina colombiana. Rev Med. 2007;15:154-62.

54. Villegas A, Botero JF, Arango IC, Arias S, Toro MM. Prevalencia del síndrome metabólico en El Retiro, Colombia. Iatreia. 2003;16:291-7.

55. Ramírez CJ, Jaramillo C. Síndrome metabólico y factores de riesgo cardiovascular en pacientes con un primer evento coronario. Acta Med Colomb. 2003;28:15-22.

56. Alayón AN, Alvear C. Prevalencia de desórdenes del metabolismo de los glúcidos y perfil del diabético en Cartagena de Indias (Colombia), 2005. Salud Uninorte. 2006;22:20-8.

57. Bautista LE, Oróstegui M, Vera LM, Prada GE, Orozco LC, Herrán OF. Prevalence and impact of cardiovascular risk factors in Bucaramanga, Colombia: Results from the Countrywide Integrated Noncommunicable Disease Intervention Programme (CINDI/CARMEN) baseline survey. Eur J Cardiovasc Prev Rehabil. 2006;13:769-75.

58. Lombo B, Villalobos C, Tique C, Satizábal C, Franco CA. Prevalencia del síndrome metabólico entre los pacientes que asisten al servicio Clínica de Hipertensión de la Fundación Santa Fe de Bogotá. Rev Col Cardiol. 2006;12:472-8.

59. Pinzón JB, Serrano NC, Díaz LA, Mantilla G, Velasco HM, Martínez LX, et al. Impacto de las nuevas definiciones en la prevalencia del síndrome metabólico en una población adulta de Bucaramanga, Colombia. Biomédica. 2007;27:172-9.

60. Guevara FO, Cañón B, Liévano MC, Lombo B, Rendón I, Blanco F. Prevalencia de síndrome metabólico en pacientes infectados con VIH: Utilizando los criterios del ATP III y de la IDF. Acta Med Colomb. 2008;33:282-8.

61. Sánchez F, Jaramillo N, Vanegas A, Echeverri JG, Alviar CL, Echavarría $\mathrm{E}$, et al. Prevalencia y comportamiento de los factores de riesgo del síndrome metabólico según los diferentes intervalos de edad, en una población femenina del área de influencia de la Clínica Las Américas, en Medellín-Colombia. Rev Colomb Cardiol. 2008;15:102-10.

62. Manzur F, de la Ossa M, Trespalacios E, Abuabara Y, Lujan M. Prevalencia de síndrome metabólico en el municipio de Arjona, Colombia. Rev Colomb Cardiol. 2008;15:215-22.

63. Manzur F, Alvear C, Alayón A. Caracterización fenotípica y metabólica del síndrome metabólico en Cartagena de Indias. Rev Colomb Cardiol. 2008;15:97-101.

64. Arteaga-Arredondo LF, Fajardo-Rodríguez HA. Prevalencia de factores de riesgo cardiovascular en pilotos de aviación civil en Colombia en el año 2005. Rev Salud Publica. 2010;12: 250-6.
65. Alayón AN, Ariza S, Baena K, Lambis L, Martínez L, Benítez L. Búsqueda activa y evaluación de factores de riesgo cardiovascular en adultos jóvenes, Cartagena de Indias, 2007. Biomédica. 2010;30:238-44.

66. Patiño-Villada FA, Arango-Vélez EF, Quintero-Velásquez MA, Arenas-Sosa MM. Factores de riesgo cardiovascular en una población urbana de Colombia. Rev Salud Publica. 2011;13:433-45.

67. Davila EP, Quintero MA, Orrego ML, Ford ES, Walke H, Arenas $M M$, et al. Prevalence and risk factors for metabolic syndrome in Medellin and surrounding municipalities, Colombia, 2008-2010. Prev Med. 2013;56:30-4.

68. Mora G, Salguedo G, Ruíz M, Ramos E, Alario A, Fortich A, et al. Concordancia entre cinco definiciones de síndrome metabólico. Cartagena, Colombia. Rev Esp Salud Publica. 2012;86: 301-11.

69. Ochoa-Orozco S, Moreno-Gutiérrez P, Echeverry-Cataño LF, Orozco-Escobar A, Mondragón-Cardona A, Villegas-Rojas S. Riesgo cardiovascular y de DM en población carcelaria de Pereira, Colombia, 2010. Rev Med Risaralda. 2012;18: 129-33.

70. Cardona-Arias JA, Rivera-Palomin Y, Llanes-Agudelo OM. Prevalencia de DM y dislipidemias en indígenas del resguardo Cañamomo-Lomaprieta, Colombia. Investigaciones Andinas. 2012;14:414-26.

71. Machado-Alba JE, Machado-Duque ME. Prevalencia de factores de riesgo cardiovascular en pacientes con dislipidemia afiliados al sistema de salud en Colombia. Rev Peru Med Exp Salud Publica. 2013;30:205-11.

72. Suarez-Flórez LE, Chavarriaga-Ríos MC. Prevalencia de DM auto reportada y factores asociados en los adultos de 60-64 años de Medellín y área metropolitana, participantes en la encuesta nacional de demografía y salud y la encuesta de situación nutricional en Colombia del año 2010. CES Salud Pública. 2014;5:21-37.

73. Cataño-Bedoya JU, Duque-Botero J, Naranjo-González CA, Rúa-Molina DC, Rosique- Gracia J, García-Pineda AF, et al. Prevalencia de factores de riesgo cardiovascular en indígenas embera-chamí de Cristianía (Jardín), Antioquia. Iatreia. 2015;28:5-16.

74. Barceló A, Rajpathak S. Incidence and prevalence of DM in the Americas. Rev Panam Salud Publica. 2001;10:300-8.

75. Karvonen M, Viik-Kajander MV, Moltchanova E, Libman I, LaPorte R, Tuomilehto J. Incidence of childhood type $1 \mathrm{DM}$ worldwide. DM Mondiale (DiaMond) Project Group. DM Care. 2000;23:1516-26.

76. Agudelo-Ochoa GM, Arias-Arteaga R. Prevalencia del síndrome metabólico en niños y adolescentes escolarizados del área urbana de la ciudad de Medellín. Iatreia. 2008;21:260-70.

77. Feliciano-Alfonso JE, Mendivil CO, Sierra-Ariza ID, Pérez CE. Cardiovascular risk factors and metabolic syndrome in a population of young students from the national university of Colombia. Rev Assoc Med Bras. 2010;56:293-8.

78. Suárez-Ortegón MF, Ramírez-Vélez R, Mosquera M, Méndez F, Aguilar-de Plata C. Prevalence of metabolic syndrome in urban Colombian adolescents aged 10-16 years using three different pediatric definitions. J Trop Pediatr. 2013;59:145-9.

79. Centro Latinoamericano y Caribeño de Demografía (CELADE) - División de Población de la CEPAL [consultado 1 Nov 2015]. Disponible en: www.eclac.cl/celade/depualc/.

80. Informe sobre Desarrollo Humano 2013. El ascenso del Sur: Progreso humano en un mundo diverso. Copyright 2013 del Programa de las Naciones Unidas para el Desarrollo 1 UN Plaza, Nueva York, NY 10017, Estados Unidos.

81. Observatorio Demográfico América Latina y el Caribe (2009). Urbanización en perspectiva. Año IV, No. 8, Octubre 2009. 
82. CEPAL. Balance Preliminar de las Economías de América Latina y el Caribe-2013. Washington DC: CEPAL; 2014.

83. Lopez-Jaramillo P, Gomez-Arbelaez D, Sotomayor-Rubio A, Mantilla-Garcia D, Lopez-Lopez J. Maternal undernutrition and cardiometabolic disease: A Latin American perspective. BMC Medicine. 2015;13:41.

84. Ng M, Fleming T, Robinson M, Thomson B, Graetz N, Margono C, et al. Global, regional, and national prevalence of overweight and obesity in children and adults during 1980-2013: A systematic analysis for the Global Burden of Disease Study 2013. Lancet. 2014;384:766-81.
85. Colberg SR. Exercise and Diabetes: A Clinician's Guide to Prescribing Physical Activity. 1st ed Alexandria, VA: American Diabetes Association; 2013.

86. Colberg SR, Sigal RJ, Fernhall B, Regensteiner JG, Blissmer BJ, Rubin RR, et al. American College of Sports Medicine; American Diabetes Association. Exercise and type 2 diabetes: The American College of Sports Medicine and the American Diabetes Association: Joint position statement executive summary. Diabetes Care. 2010;33:2692-6. 